\title{
Antenna Arrangement Verification for Low Sidelobe Levels
}

\author{
Abigail J. Kragt Finnell, Peter J. Schubert \\ Indiana University-Purdue University Indianapolis, Indianapolis, Indiana, USA
}

\begin{abstract}
Space-to-earth Wireless Power Transfer (WPT) in large scale will not be allowed unless the side lobe levels (SLL) can be reduced many orders of magnitude lower than the current technology allows. In particular, high SLL could potentially interfere with aircraft communications around the beam, while the area inside the beam would necessarily be a no-fly zone, similar as over nuclear power plants. To overcome this, the transmitting antenna must be cleverly designed and controlled. In this work, independent validation of the layout, spacing, and envelope arrangement of a design first proposed in 2016 is performed and presented. This design involves a hexagonal design with a triangular antenna element arrangement and a spacing of 0.8 wavelengths using the Dolph-Chebychev beam profile. While this has been shown to produce $-240 \mathrm{~dB}$ SLL in the AWR Design Environment already, it will now be analyzed using the MATLAB Phased Array System Toolbox. The design will also be investigated on a smaller scale, with the potential for use in other applications, including the powering of low orbit weather balloons or unmanned aerial vehicles (UAVs). The possibility of very low SLL would be transformational in these and other WPT applications, including space solar power, and could greatly benefit humanity and the environment.
\end{abstract}

Index Terms-phased array antenna, side lobe level, off-axis energy, desensitization, space solar power

\section{INTRODUCTION}

W IRELESS POWER TRANSFER (WPT) relies on directionality to transmit from a source to a receiver. While off-axis power is at least inefficient, in the case of very high power levels, such stray energies are anathema. In the case of Space Solar Power ${ }^{3,8,11,12}$ (SSP) with a beamwidth measured in kilometers and beam spread measured in arcseconds desensitization of other wireless applications can disrupt the radios of first responders, scramble cell phones, and jam aircraft communications. Such a situation is untenable, and cannot conceivably be allowed by society, regardless of the many benefits of SSP. Textbooks and technical literature on sidelobe levels have not improved much since the early days in the 1930s where first sidelobe levels (SLL) of phased array antennas (PAA) have power densities which are $-20,-30$, or $-40 \mathrm{~dB}$ down from the primary lobe ${ }^{6}$. Methods such as truncated Gaussian, Taylor, of Dolph-Chebychev profiles of the power level fed to the center antenna element of a transmit PAA to those elements at the periphery of the array have been studied, but until recently there were no reported SLL better than (lower than) $-60 \mathrm{~dB}^{1,2}$.

This work was sponsored by the Richard G. Lugar Center for Renewable Energy (www.lugarenergycenter.org), administratively housed within the Purdue School of Engineering and Technology, IUPUI, Indianapolis, IN, USA.
At that level, a $5 \mathrm{GW}$ power beam will desense an entire continent.

In 2016 it was discovered, using a rf design tool, that a PAA having a triangular arrangement of elements at 0.8 wavelength spacing within a hexagonal boundary having 6000 elements across can use the Dolph-Chebychev SLL minimization and achieve sidelobe levels as much as $-240 \mathrm{~dB}$ down from the main lobe $e^{4,7}$. Such a large transmit antenna is appropriate for SSP, but is quite large compared to existing PAA designs. A primary objective of this paper is to validate this result using a different rf design tool. In addition, this work will study the degree to which such low SLL can be achieved as the transmit antenna is scaled down from SSP size to more modest scale applications such as emergency response, drone recharging, aerostat powering, building-to-building power sharing, and WPT to recharge a moving electric vehicle.

\section{METHODS}

The main focus of this research is on a triangular lattice antenna arrangement within a hexagonal perimeter based on a paper by one of the authors published in 2016. This arrangement, along with a Dolph-Chebychev beam profile, produced SLL that were well below $-60 \mathrm{~dB}$ for transmit antenna dimensions consistent with SSP. The ability to dial down the SLL using the established radio frequency (rf) tool AWR (National Instruments) was a surprising discovery. The effect is not obvious in the design of smaller arrays. These findings suggest two needed areas for further research: (1) validate the extremely low SLL with an independent rf design tool; and (2) determine at what minimum array size the onset of extremely low SLL can be effective.

The first work, of validation, was performed with the Phased Array Toolbox of MATLAB (The MathWorks, Natick, MA). Although the two rf tools do not have the exact same capabilities, both were used to find a common intersection in which the validation study could be performed. For all studies the S-band frequency of $2.45 \mathrm{GHz}$ was used, the antenna elements were placed on an equilateral triangle unit cell for the array, and the spacing between elements was 0.8 wavelengths, or $9.76 \mathrm{~cm}$ (3.8 inches). Array configurations with either hexagonal or rectangular outer envelope shapes were studied.

Corresponding author: Schubert (pjschube@iupui.edu) 
In all cases, the distribution of power followed a DolphChebychev power profile, with independent $\mathrm{X}$ and $\mathrm{Y}$ amplitude schedules multiplied by the appropriate trigonometry for a $2 \mathrm{D}$ distribution.

\section{A. AWR Design Environment}

The antenna arrangement in the AWR Design Environment involved antenna elements with a gain of $14.4 \mathrm{~dB}$ and a power of $23 \mathrm{dBm}$. The elements were arranged in a triangular lattice that was 107 elements tall and 107 elements wide.

A Dolph-Chebychev beam profile was used, with a SLL reduction of $120 \mathrm{~dB}$. The resulting beam profile, as shown in Figure 1, had a SLL reduction of around $-140 \mathrm{~dB}$.

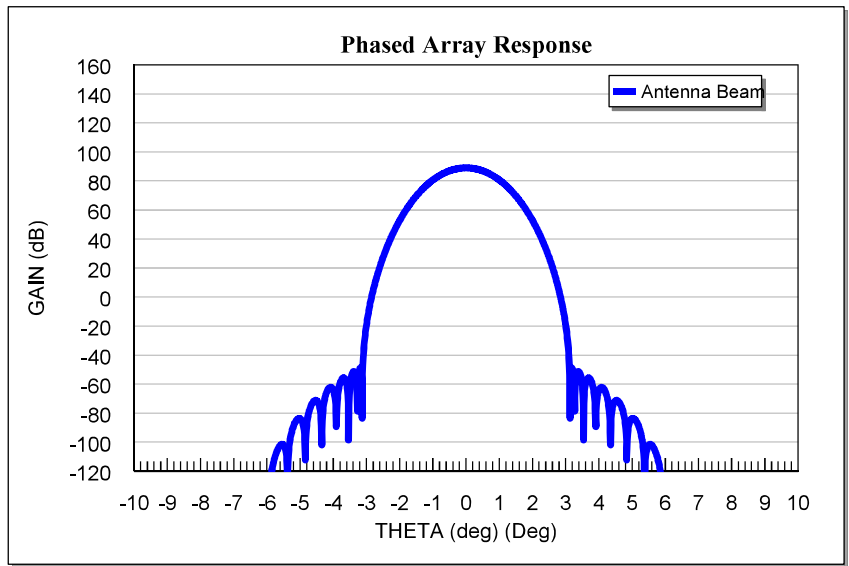

Fig. 114.4 dB Antenna Gain

An arrangement with a $0 \mathrm{~dB}$ antenna gain was also analyzed, simply to ensure that the MATLAB and AWR antenna arrangements were set up as similarly as possible. This produced a similar result as shown in Figure 2, with a SLL reduction of around $-140 \mathrm{~dB}$.

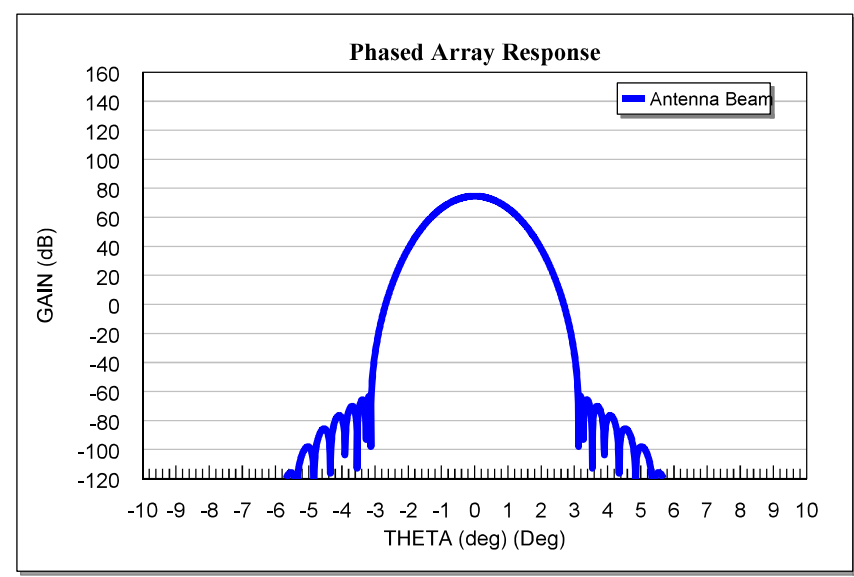

Fig. $20 \mathrm{~dB}$ Antenna Gain

\section{B. MATLAB}

The Phased Array Toolbox in MATLAB was used to evaluate an antenna arrangement that was as close as possible to the previously mentioned array. It was also 107 by 107 elements wide and in a triangular arrangement, using the
phased.URA tool.

This program implemented a Dolph-Chebychev beam profile as well. This was done by creating a Dolph-Chebychev beam profile for 107 linear elements, and then applying the taper to each element of the array by multiplying the previously created linear taper to itself to create a 2D taper array.

This arrangement was evaluated in two different ways, the first being a hexagonal arrangement, as shown in figure 3. This was done to match the AWR arrangement. The taper pattern of the array is as shown.

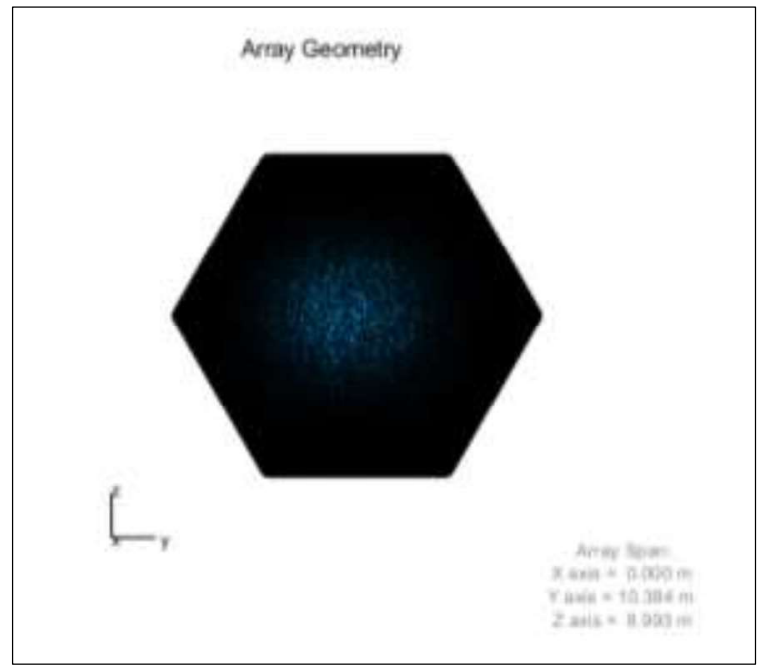

Fig. 3 Hexagonal Perimeter Dolph-Chebychev Taper

The result of this arrangement, shown in figure 4, did not show as much of a reduction in SLL as in AWR, only around $-60 \mathrm{~dB}$. Although this is not a bad result, as it is around the level reported before 2016, it is not low enough for space-toearth power transfer on a large scale, as desired.

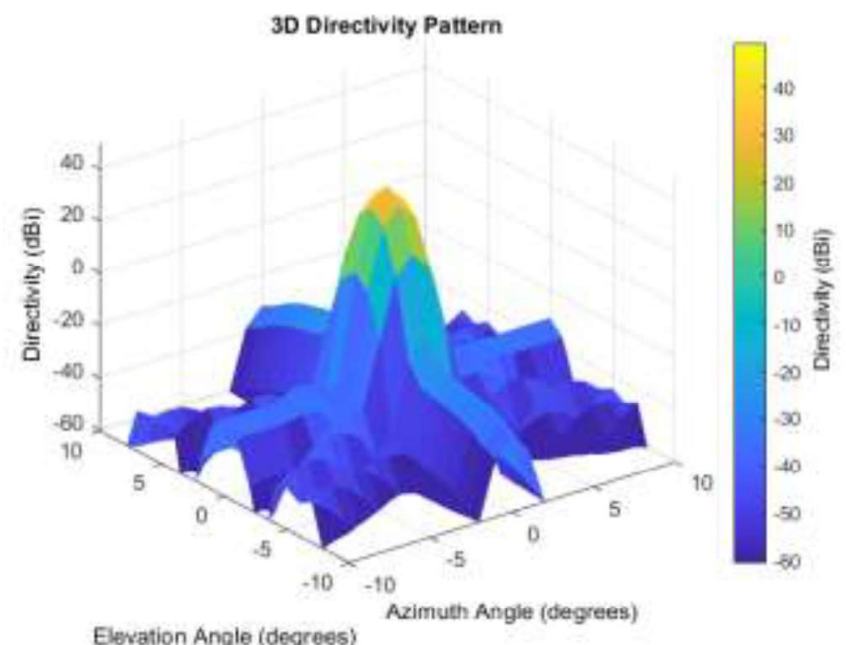

Fig. 4 Hexagonal Perimeter Beam Pattern

Also evaluated was an arrangement of antennas in a triangular lattice with a square perimeter, as shown in figure 5. Although it is not quite the same as the construction in 
AWR, it had the surprising result of side-lobe reduction at a much larger scale, around $-120 \mathrm{~dB}$, the results of which are shown in Figures 6 and 7.

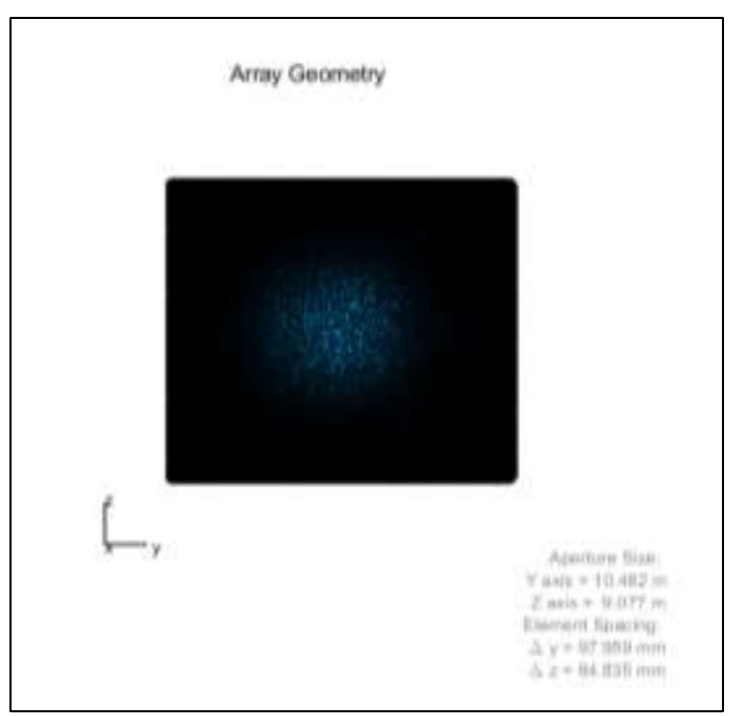

Fig. 5 Square Perimeter Dolph-Chebychev Taper

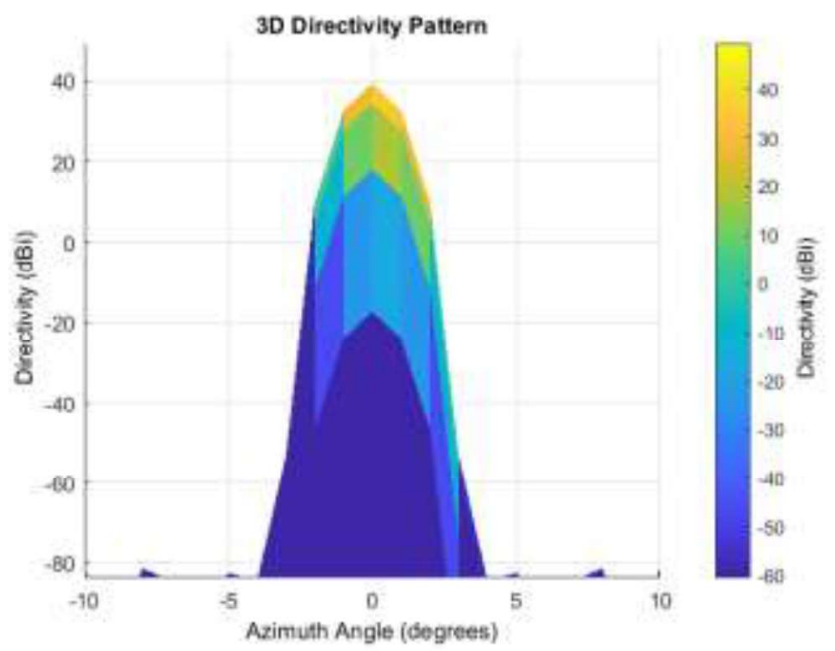

Fig. 6 Square Perimeter Beam Pattern vs. Azimuth Angle

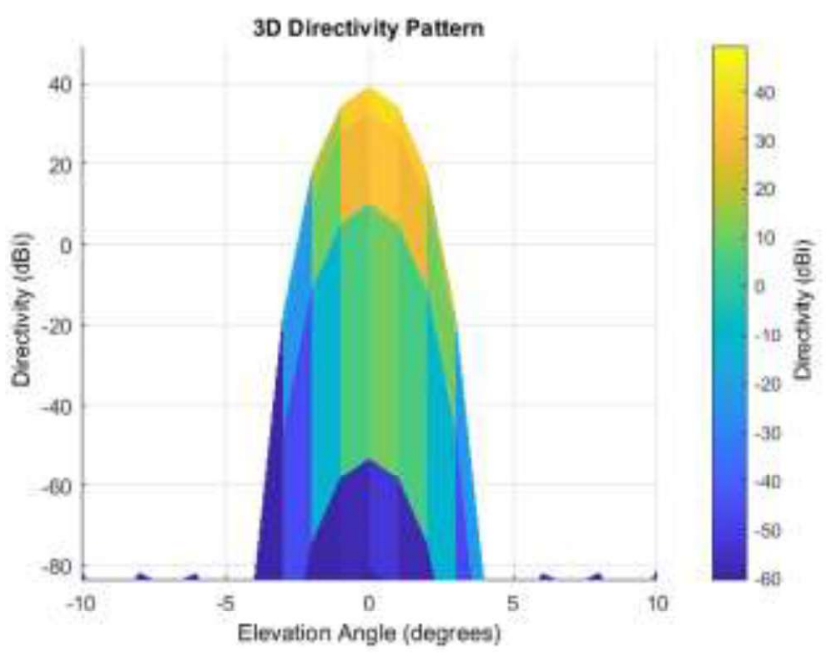

Fig. 7 Square Perimeter Beam Pattern vs. Elevation Angle

The square arrangement beam pattern shows a reduction in side lobe levels of around $-120 \mathrm{~dB}$, which is similar to the $0 \mathrm{~dB}$ antenna beam pattern produced by AWR.

\section{Minimum Array Size}

The minimum array size for SLL reduction was found by testing increasingly small array sizes to find the eventual decrease in SLL reduction.

In AWR, each decrease in the number of elements only increased the width of the main beam, while the SLL reduction remained fairly consistent at around $-130 \mathrm{~dB}$. In MATLAB, using the square perimeter arrangement, this was also the case, with increased main beam size and SLL constant at around $-120 \mathrm{~dB}$.

In order to find a realistic small-antenna size, the main beam width was kept between -5 and 5 degrees; with this condition, the number of elements in AWR for each side was 75, with the results shown in Figure 8.

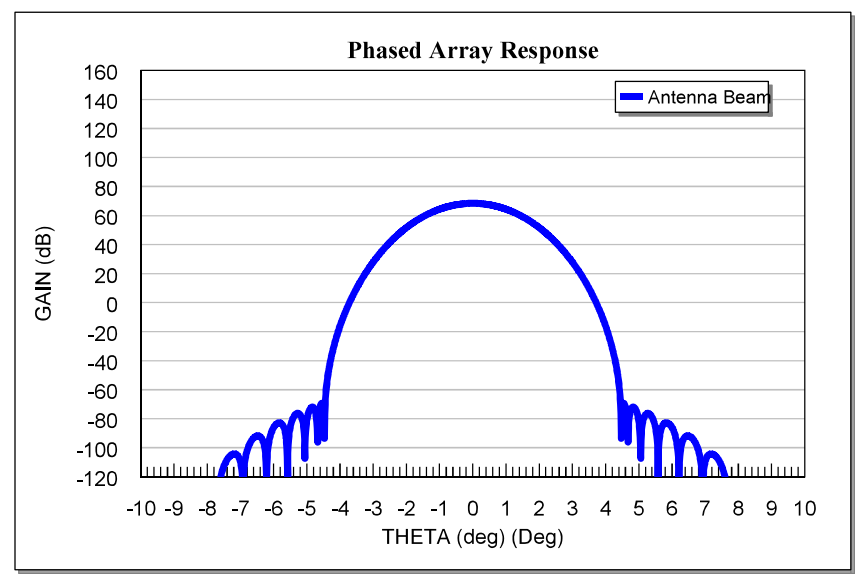

Fig. 875 by 75 Element, 0 dB Antenna Beam Profile

Additionally, this same number of elements in MATLAB also showed a main beam within the -5 to 5 degree limit, as shown in Figure 9. 


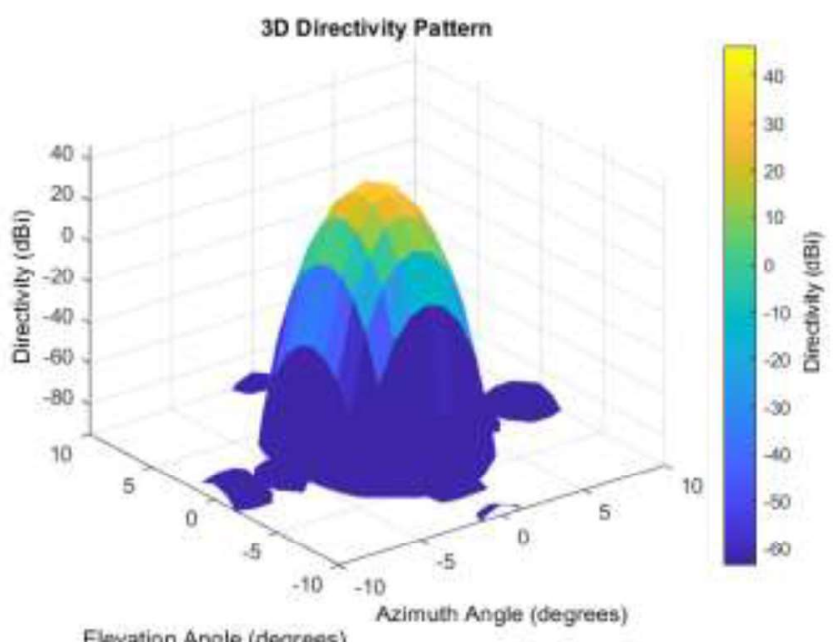

Fig. 975 by 75 Element Square Perimeter Beam Pattern

\section{RESULTS}

The purpose of this analysis held two parts; one, to verify that a SLL below $-60 \mathrm{~dB}$ is possible with a triangular lattice arrangement and Dolph-Chebychev beam profile, and two, to identify the smallest allowable antenna arrangement that retains this large side lobe level reduction for the purpose of terrestrial power applications.

The reduction in SLL shown in AWR was verified in MATLAB by the triangular lattice arrangement in a square perimeter. Although there is a small discrepancy of less than 20 $\mathrm{dB}$ between the $0 \mathrm{~dB}$ gain AWR result and the square perimeter MATLAB result, this is most likely the result of the different assumptions and information given to each design tool. With further study, a verification involving the specific type of antenna element, including a $14.4 \mathrm{~dB}$ gain, could be found.

The reduction in number of antenna elements also showed a favorable response. In both software programs, a reduction to the size of 75 by 75 elements kept the same SLL reduction with a main beam within -5 to 5 degrees. This allows for an antenna arrangement only around $7.2 \mathrm{~m}$ in its largest dimension, which is less than the size of a typical highway billboard.

\section{DISCUSSION}

The results of this research are very promising. This research paves the way for future work in spacetennae for space-to-earth applications. In future research, an increased number of elements in MATLAB would be necessary to show decreased width of the beam, as would be expected for an array of larger size.

Another future area of interest is in the specification of element type in MATLAB. This would help verify the previous work done in AWR with $14.4 \mathrm{~dB}$ antenna elements.

The results of the reduction in size for an antenna arrangement is also very promising for other applications in the near future. The use for a small-scale wireless power transfer device at middle distances is vast; not only could this apply to drones or other aerial devices; it could potentially allow for energy sharing between neighbors or in difficult-to-get-to areas.

\section{CONCLUSIONS}

A new discover in the reduction of side lobe level below -60 $\mathrm{dB}$, first shown by Schubert in 2016, has been verified. The MATLAB and AWR Design Environment layouts agree that for a $2.45 \mathrm{GHz}$ frequency, with a 0.8 wavelength spacing and in a triangular lattice, and with a Dolph-Chebychev beam profile, applied in both the $\mathrm{X}$ and $\mathrm{Y}$ directions, a reduction in SLL greater than $-110 \mathrm{~dB}$ is possible.

Additionally, a reduction in size of antenna elements maintains the low side lobe level, while at the expense of increasing the width of the main beam. For a main beam within the limits of -5 to $5 \mathrm{~dB}$, both AWR and MATLAB designs agree that an arrangement of at least 75 by 75 elements is required to observe this newly-reported effect.

\section{REFERENCES}

[1] Maillot, R.J., Phased Array Antenna Handbook, Artech House, Inc., 1994, Norwood, MA.

[2] Elliott, R.S., Antenna Theory and Design, J. Wiley \& Sons, 2006.

[3] Department of Energy and NASA, "Satellite Power System Concept Development and Evaluation Program, Reference System Report," Oct. 1978.

[4] McSpadden, J., "Solar Power Satellite Technology and Frequency Selection," IEEE Wireless Power Transfer Conference 2015, Boulder, CO, 13-15 May 2015.

[5] T. Hatsuda, K. Ueno, and M. Inoue, "Solar power satellite interference assessment," IEEE Microwave Magazine, vol. 3, no. 4, pp. 65-70, Dec. 2002.

[6] Dolph, C.L, "A Current Distribution for Broadside Arrays Which Optimizes the Relationship Between Beam Width and Side-Lobe Level," Proc. IRE and Waves and Electrons, pp. 335-348, June 1946.

[7] Schubert, P.J., "Sidelobe Reduction for GEO to Earth Wireless Power Transfer," Int'l. Astro. Conf. 2016, Guadalajara, Jalisco, Mexico, 26-29 Sept 2016.

[8] Love, A.W., "Basics of SPS Power Transmission from Space," IEEE Antennas and Prop. Soc. News., pp. 5-8, Dec. 1980.

[9] FCC Part 18 Rules, http://www.ecfr.gov/cgi-bin/textidx?SID $=04 f 40821 \mathrm{a} 974719865204 \mathrm{a} 7 \mathrm{~d} 9 \mathrm{f} 9 \mathrm{cce} 83 \& \mathrm{mc}=$ true \&node=pt47.1.18\&rgn=div5, accessed 9 Aug 2016.

[10] Goubau, G., Schwering, F., "On the Guided Propagation of Electromagnetic Wave Beams," IRE Trans. Antennas and Prop., pp. 248-256, May 1961.

[11] Lund, Jr., WW., and Rathjen, S.M., "Microwave Phased Array Design Considerations for SPS," Proceedings, Am. Inst. of Aero., v.1, 20-25 Aug 1978, pp 201-204, copyright 1978 Soc. of Auto. Engr, Inc. SAE/P-78/75.

[12] Denman, O.S., Gewin, R.J., Lund, Jr. W.W., Nalos, E.J., Rathjen, S.M., "A Microwave Power Transmission System for Space Satellite Power," Proceedings, Am. Inst. of Aero., v.1, 20-25 Aug 1978, pp 162-168, copyright 1978 Soc. of Auto. Engr, Inc. SAE/P-78/75. 